\title{
Relative Abundance of Insect Pests and Pod Damage by Pod Borers in Short duration Pigeonpea under Intercropping Systems
}

\author{
R. Shravan Kumar ${ }^{1 *}$, Paras Nath ${ }^{2}$ and S.V.S. Raju ${ }^{2}$ \\ ${ }^{1}$ Regional Agricultural Research Station, PJTSAU, Warangal- 506007, Telangana, India \\ ${ }^{2}$ Department of Entomology and Agricultural Zoology, Institute of Agricultural Sciences, \\ Banaras Hindu University, Varanasi, Uttar Pradesh, India \\ *Corresponding author
}

\begin{tabular}{|l|}
\hline K e y w o r d s \\
Arthropod diversity, \\
$\begin{array}{l}\text { Short duration pigeonpea, } \\
\text { Intercropping systems, } \\
\text { Relative abundance, Pod } \\
\text { damage, Seed damage }\end{array}$ \\
\hline Article Info \\
\hline $\begin{array}{l}\text { Accepted: } \\
\text { 04 November } 2018 \\
\text { Available Online: } \\
\text { 10 December } 2018\end{array}$ \\
\hline
\end{tabular}

\section{Introduction}

Pigeonpea (Cajanus cajan (L.) Millsp. is an important pulse crop which is the most widely

\section{A B S T R A C T}

A research work to study the richness of arthropod diversity under short duration pigeonpea intercropping systems was carried out at Agricultural Research Farm of Banaras Hindu University, Varanasi (UP). The faunal composition of insect pests associated with short duration pigeonpea intercropping systems in kharif 2004 and 2005 was of eight orders viz., Orthoptera, Homoptera, Thysanoptera, Lepidoptera, Diptera, Hymenoptera and Coleoptera. One bird pest of order Psittaciformes was also recorded. The twenty species of insect pests and one bird pest species were recorded infesting short duration pigeonpea under intercropping systems. The insect pests observed at vegetative and reproductive stages of the crop growth were green jassid, tur pod bug, green stink bug, gram pod borer and blue butterfly. The remaining insect pests were active either at vegetative or reproductive stage of the crop. The rose ringed parakeet was observed feeding pods at reproductive stage of crop growth. The relative abundance in the pigeonpea + sorghum $\left(\mathrm{T}_{1}\right)$ treatment plot was highest in case of flower thrips ( $M$. usitatus) and lowest in case of green stink bug. In sole crop of pigeonpea higher relative abundance was recorded by scale insects and lower by leaf cutter bees. Among all insect pests and in all treatments flower thrips had highest relative abundance and green stink bug had recorded lowest relative abundance during both the years of experimentation. The relative abundance of insect pests was very much low in pigeonpea + sorghum and highest in sole crop of pigeonpea. The average of two years data on the extent of pod damage by gram pod borer showed that pod damage $(\%)$ was more in sole crop of pigeonpea $\left(\mathrm{T}_{7}\right)$ while less in pigeonpea + sorghum $\left(\mathrm{T}_{1}\right)$. The data on pod damage $(\%)$ by tur pod fly recorded during both the years was pooled to know the overall effect of intercropping on pod damage (\%) and it was found that pod damage $(\%)$ was significantly reduced in pigeonpea + sorghum $\left(\mathrm{T}_{1}\right)$ than in other treatments. Seed damage $(\%)$ due to gram pod borer and pod fly was more in pigeonpea grown alone while it was less in the intercrops pigeonpea $+\operatorname{sorghum}\left(\mathrm{T}_{1}\right)$. grown crop in the country and has been under cultivation for over 3000 years. According to Vavilov (1951), India is the origin place of pigeonpea. It is an important pulse or grain 
legume crop in semi-arid tropical and subtropical areas of the world. Traditionally long duration varieties of pigeonpea which take about 240-270 days to mature are of low yield potential and susceptible to diseases and pests. These varieties are also damaged by frost which frequently occurs in most parts of northern India in the month of December and January. Recently some short duration varieties of about 130-160 days have been developed which have high yield potential (20-30 q/ha) and harvested by the end of November. These varieties fit very well under double cropping systems with wheat and other Rabi crops.

There is an immense scope for short duration pigeonpea in the future and there is a dearth of information regarding insect pests on flowers and pods of short duration pigeonpea. Therefore, a study was carried out with the objective of study of the richness of Arthropod diversity and pod and seed damage (\%) by Lepidopteran borers and pod fly under short duration pigeonpea intercropping systems.

\section{Materials and Methods}

A field experiment was carried out during kharif season of 2004 and 2005 at the Agricultural Research Farm, Banaras Hindu University, Varanasi, Uttar Pradesh.

The following crops were grown as intercrops with short duration pigeonpea so as to assess the richness of arthropod diversity associated with foliage, flowers and pods of pigeonpea.

The short duration pigeonpea variety UPAS120 was grown during the kharif season of 2004 and 2005 in the well prepared field on raised flat bed made by flat bed planter at a spacing of $60 \times 20 \mathrm{~cm}$ between row to row and plant to plant, respectively. No plant protection measures were exercised during the period of experiment.

\section{Observations}

Qualitative and quantitative composition of insect pests

During the period of experimentation the qualitative and quantitative composition of Arthropods (insect pests) associated with foliage, flowers and pods of pigeonpea plants was recorded. The insect pests in the experimental plots of pigeonpea intercropping systems were collected and identified starting from the seedling stage onwards. The population of major pests was recorded by observing randomly selected five plants from three middle rows. The jassid (Empoasca kerri) population was recorded by observing 10 trifoliate leaves selected from 5 randomly selected plants of three middle rows. The population of flower thrips (Megalurothrips usitatus) was taken from randomly selected 10 flowers picked up at random from each plot. The florets were dissected under magnifying glass and the number of thrips was counted. The population of bud weevil (Indosocladius asperulus) was recorded out of ten flowers picked up from 5 plants of 3 middle rows of each plot. The population of pod borer (Helicoverpa armigera), legume pod borer (Maruca testulalis), plume moth (Exelastis atomosa), pod sucking bug (Clavigralla gibbosa), blue butterfly (Lampides boeticus), blister beetle (Mylabris pustulatus) and leaf webber (Grapholita critica) was recorded on 5 randomly selected plants from 3 middle rows of each plot. The pod fly larval population was recorded by observing 10 pods picked up from the 5 randomly selected plants from 3 middle rows of each plot. The collected pods were opened and the population was recorded. The population counts of larvae of insect pests except thrips and pod fly was made by observing three middle rows from each plot in the morning hours. The richness of Arthropod diversity (insect pests) associated with foliage, flowers and pods of short duration pigeonpea 
and the intercrops (the qualitative and quantitative composition) was recorded at weekly interval. The data recorded was used to analyze the relative abundance and richness of species diversity. No insecticidal spray was carried out in this set of experiment.

\section{Percent pod damage}

After the maturity of the crop 150 pods from each treatment were collected from the middle portion of each plot. From these selected pods healthy and damaged pods were sorted out separately for computing the damage percentage by means of the formula stated below (Sahoo et al., 2000).

Pod Damage $(\%)=$

No. of damaged pods

Total No. of seeds collected

\section{Per cent seed damage}

The seed damage done by Lepidopteran borers and pod fly was assessed. The seeds collected by opening 150 pods were observed for recording healthy and damaged grains and the data thus obtained were subjected by Sahoo et al., 2000.

\section{No. of damaged seeds \\ Pod Damage $(\%)$ = ------------------ x 100 \\ Total No. of seeds collected \\ Qualitative and quantitative composition of natural enemies of pigeonpea insect pests}

The observations were recorded on the qualitative and quantitative composition of natural enemies of insect pests associated with the pigeonpea intercropping system.

\section{Data computation}

The population of insect pest and their natural enemies associated with pigeonpea under intercropping system recorded at weekly intervals were analysed to know the trend of population fluctuation. The data were used to compute the relative abundance of insect pests and their natural enemies.

The relative abundance (dominance or relative density or R.A) was computed from the data as described by Fager (1957) and Wallwork (1976) by using following formula:

Relative Abundance $(\%)=\stackrel{a}{---} \times 100$

$\mathrm{n}$

Where ' $a$ ' is the number of individuals in a sampling unit and ' $n$ ' is the total number of individuals in all the units.

\section{Results and Discussion}

The composition of Arthropod pests associated with the short duration pigeonpea based intercropping was constituted by eight orders insects viz., Orthoptera (1 family), Homoptera (2 families), Hemiptera (3 families), Thysanoptera (1 family), Lepidoptera (5 families), Diptera (1 family), Hymenoptera (1 family) and Coleoptera (3 families). One bird pest, rose ringed parakeet was also recorded (Table 1).

Qualitative and quantitative composition of insect pests and bird pests infesting short duration pigeonpea under intercropping systems at Varanasi

Qualitative composition of pests associated with short duration pigeonpea showed twenty species of insect pests belonging to eight orders and seventeen families and one bird pest. The family Acrididae was represented by one species. The family Cicadellidae was represented by one species. Families Membracidae and Coccidae were represented by one species each. The families Coreidae 
and Pentatomidae were represented by two species each. The families Noctuidae, Tortricidae, Lycaenidae, Pterophoridae, Pyralidae of order Lepidoptera were represented by one species each. The families Apionidae, Meloidae of Coleoptera order were represented by one species each. The family Curculionidae was represented by two species. One bird pest was also recorded belonging to family Psittacidae of order Psittaciformes.

The twenty species of insect pests encountered in short duration pigeonpea were grasshopper (Attractomorpha crenulata), green jassid (Empoasca kerri), cow bug (Otinotus oneratus), tur pod bug (Clavigralla gibbosa), scale insect (Ceroplastodes cajani), pod bug (Riptortus pedestris), lab lab bug (Coptosoma cribrarium), green stink bug (Nezara viridula), gram caterpillar (Helicoverpa armigera), leaf webber (Grapholita critica), blue butterfly (Lampides boeticus), plume moth (Exelastis atomosa), legume pod borer (Maruca testulalis), tur pod fly (Melanagromyza obtusa), flower thrips (Megalurothrips usitatus), leaf cutter bees (Megachile sp), pod weevil (Apion benignum), leaf damaging weevil (Myllocerus undecimpustulatus), blister beetle (Mylabris pustulatus), bud weevil (Indozocladius asperulus). One bird pest species, rose ringed parakeet (Psittacula krameri) was also recorded (Table 2).

Grasshopper (A. crenulata), cow bug ( $O$. oneratus), leaf cutter bees (Megachile sp), ash weevil (M. undecimpustulatus), lab lab bug (C. cribrarium) and leaf webber (G. critica) were present during the vegetative stage of the crop growth. Pod bug (R. pedestris), plume moth (E.atomosa), legume pod borer ( $M$. testulalis), tur pod fly (M. obtusa), flower thrips (M. usitatus), pod weevil (A. benignum), blister beetle (M. pustulatus) and bud weevil (I. asperulus) were observed at reproductive stage of the crop growth. The insect pests observed at both vegetative and reproductive stage of the crop growth were viz., green jassid (E.binotata), tur pod bug (C. gibbosa), green stink bug (N. viridula), gram caterpillar (H. armigera) and blue butterfly (L. boeticus). Rose ringed parakeet (P.krameri) was observed feeding pods at reproductive stage of the crop growth. Parakeet's damage was more to those pigeonpea plants where perching site such as trees, electric wires and their poles were situated nearby were used as perches by the rose ringed parakeet for perching. The observations of various insect pests infesting short duration pigeonpea are similar to those of Kumar and Nath (2004), who reported that UPAS-120 was severely infested by Lampides boeticus, Megalurothrips usitatus, Mylabris pustulata, Exelastis atomosa, Maruca testulalis, Indigocladices asperulus, Melanagromyza obtusa and Clavigralla gibbosa. Kumar and Nath (2004) who reported that, pigeonpea variety, UPAS-120 was infested by twenty six species belonging to two classes of the Animalia kingdom i.e. Insecta and Aves atvarious stages of crop growth in an overlapping manner right from seedling to harvesting stage of crop growth.

Treatment wise relative abundance of insect pests observed in the years 2004 and 2005 cropping seasons is shown in Table 3 and 4. In 2004 , in pigeonpea + sorghum $\left(\mathrm{T}_{1}\right)$ treatment, flower thrips showed highest relative abundance (1.78), and green stink bug showed lowest relative abundance $(0.01)$. In pigeonpea + bajra $\left(\mathrm{T}_{2}\right)$ treatment, scale insect showed higher relative abundance (2.39) and green stink bug recorded lower relative abundance (0.01). In case of pigeonpea + greengram $\left(\mathrm{T}_{3}\right)$ treatment flower thrips found with higher relative abundance (3.00) and green stink bug found with lower relative abundance (0.06). In treatments pigeonpea + cowpea $\left(\mathrm{T}_{4}\right)$ and pigeonpea + soybean $\left(\mathrm{T}_{5}\right)$ as in pigeonpea + greengram $\left(\mathrm{T}_{3}\right)$ treatment, flower thrips had higher relative abundance (3.06 and 3.35 
respectively), green stink bug had lower abundance (0.06 and 0.01 respectively). In case of pigeonpea + urdbean $\left(\mathrm{T}_{6}\right)$ treatment flower thrips had higher relative abundance (3.49) and scale insect showed lower relative abundance (0.04). In sole crop of pigeonpea $\left(\mathrm{T}_{7}\right)$, higher relative abundance was found to scale insect and lower relative abundance had been recorded by leaf cutter bee. Among all insect pests and all treatments, flower thrips had recorded highest relative abundance (21.55) and green stink bug had recorded lowest relative abundance (0.63).

In 2005, almost same trend as in 2004 was repeated. In pigeonpea + sorghum $\left(\mathrm{T}_{1}\right)$ treatment flower thrips had higher relative abundance (1.78) and green stink bug had lower relative abundance (0.00). In case of pigeonpea + bajra $\left(\mathrm{T}_{2}\right)$ treatment scale insect recorded higher relative abundance (2.30) and green stink bug had lower relative abundance (0.01). As in pigeonpea + sorghum $\left(\mathrm{T}_{1}\right)$ treatment, in pigeonpea + greengram $\left(\mathrm{T}_{3}\right)$, pigeonpea + cowpea $\left(\mathrm{T}_{4}\right)$, pigeonpea + soybean $\left(\mathrm{T}_{5}\right)$, pigeonpea + urdbean $\left(\mathrm{T}_{6}\right)$ treatments also flower thrips had higher relative abundance $(2.76,2.88,3.10$ and 3.25 resp.).

In sole crop of pigeonpea $\left(\mathrm{T}_{7}\right)$, flower thrips recorded higher relative abundance (4.27) and leaf cutter bees recorded lower relative abundance (0.30). Among all treatments and all insect pests as in 2004, in 2005 also flower thrips had recorded higher relative abundance (20.26) and green stink bug recorded lower relative abundance (0.98).

Relative abundance of insect pests was very much low in pigeonpea $+\operatorname{sorghm}\left(\mathrm{T}_{1}\right)$ and high in sole crop of pigeonpea $\left(\mathrm{T}_{7}\right)$.

Nath and Singh (1998) demonstrated that intercropping with non-host plants reduced the insect pest incidence whereas intercropping with similar plant type accentuated the pest problems further. Pimental (1961), reported that arthropod pests out breaks were more likely to occur in monocultures than in polycultures.

These results were in confirmation with results of Rao et al., (2003) who reported that castor and sorghum as intercrops with pigeonpea reduced leaf hopper infestation significantly than the sole crop.

Sekhar et al., (1997) found that when sorghum was intercropped with pigeonpea, it gave the largest reduction in jassid population followed by greengram, groundnut and rice. Pigeonpea + rice followed by pigeonpea + sorghum, sprayed with NSKE was the most effective in reducing the damage inflicted by gram pod borer (Nath and Singh, 2006).

Pest incidence was less in pigeonpea + sorghum $\left(\mathrm{T}_{1}\right)$ in this study, because as mentioned Romeis et al., (1998), the synchronized flowering times of short duration pigeonpea and sorghum would facilitate the transfer of natural enemies from sorghum to pigeonpea.

\section{Effect of intercropping on the extent of pod damage $(\%)$ by the gram pod borer $(H$. armigera) in short duration pigeonpea}

The data recorded on the extent of pod damage by the gram pod borer are presented in Table 5. Pod damage (\%) was significantly reduced in pigeonpea grown under intercropping system in comparison to sole pigeonpea.

The average of two years data on the extent of pod damage by gram pod borer showed that pod damage $(\%)$ was more in sole crop of pigeonpea $\left(\mathrm{T}_{7}\right)$ while less in pigeonpea + sorghum $\left(\mathrm{T}_{1}\right)$. 
Table.1 Arthropod and bird diversity under short duration pigeonpea intercropping systems

\begin{tabular}{|c|c|c|c|}
\hline \multicolumn{4}{|c|}{ Insect Pests } \\
\hline S. No. & Order & Family & $\begin{array}{c}\text { No. of } \\
\text { species/ } \\
\text { family }\end{array}$ \\
\hline 1 & Orthoptera & Acrididae & 1 \\
\hline \multirow[t]{2}{*}{2} & Homoptera & a. Cicadellidae & 1 \\
\hline & & b. Membracidae & 1 \\
\hline \multirow[t]{3}{*}{3} & Hemiptera & a. Pentatomidae & 2 \\
\hline & & b. Coreidae & 2 \\
\hline & & c. Coccidae & 1 \\
\hline 4 & Thysanoptera & Thripidae & 1 \\
\hline \multirow[t]{5}{*}{5} & Lepidoptera & a. Noctuidae & 1 \\
\hline & & b.Tortricidae & 1 \\
\hline & & c. Lycaenidae & 1 \\
\hline & & d. Pyralidae & 1 \\
\hline & & e. Pterophoridae & 1 \\
\hline 6 & Diptera & Agromyzidae & 1 \\
\hline 7 & Hymenoptera & Megachilidae & 1 \\
\hline \multirow[t]{3}{*}{8} & Coleoptera & a. Curculionidae & 2 \\
\hline & & b. Meloidae & 1 \\
\hline & & c. Apionidae & 1 \\
\hline 9 & Psittaciformes & Psittacidae & 1 \\
\hline
\end{tabular}


Table.2 Qualitative composition of insect pests and bird pests infesting short duration pigeonpea grown under intercropping systems at various stages of growth

\begin{tabular}{|c|c|c|c|c|c|c|}
\hline \multirow[t]{2}{*}{ S. No. } & \multirow{2}{*}{$\begin{array}{l}\text { Common name of } \\
\text { Insect pest }\end{array}$} & \multirow{2}{*}{$\begin{array}{l}\text { Scientific name of } \\
\text { Insect Pest }\end{array}$} & \multirow[t]{2}{*}{ Family } & \multirow[t]{2}{*}{ Order } & \multicolumn{2}{|c|}{ Crop stage } \\
\hline & & & & & Vegetative Stage & Reproductive Stage \\
\hline 1 & Grasshopper & $\begin{array}{l}\text { Attaractomorpha } \\
\text { crenulata }\end{array}$ & Acrididae & Orthoptera & YES & --- \\
\hline 3 & Cow bug & Otinotus oneratus & Membracidae & Homoptera & YES & --- \\
\hline 4 & Scale Insect & Ceroplastodes cajani & Coccidae & Hemiptera & YES & --- \\
\hline 5 & Tur pod bug & Clavigralla gibbosa & Coreidae & Hemiptera & YES & YES \\
\hline 7 & Lab lab bug & Coptosoma cribrarium & Pentatomidae & Hemiptera & YES & --- \\
\hline 8 & Green stink bug & Nezara viridula & Pentatomidae & Hemiptera & YES & YES \\
\hline 9 & Gram pod borer & Helicoverpa armigera & Noctuidae & Lepidoptera & YES & YES \\
\hline 10 & Leaf webber & $\begin{array}{l}\text { Grapholita (Eucosma) } \\
\text { critica }\end{array}$ & Tortricidae & Lepidoptera & YES & --- \\
\hline 11 & Blue butterfly & Lampides boetica & Lycaenidae & Lepidoptera & YES & YES \\
\hline 15 & Flower thrips & $\begin{array}{l}\text { Megalurothrips } \\
\text { usitatus }\end{array}$ & Thripidae & Thysanoptera & --- & YES \\
\hline 16 & Leaf cutter bees & Megachile sp & Megachilidae & Hymenoptera & YES & --- \\
\hline 17 & Pod weevil & Apion benignum & Apionidae & Coleoptera & --- & YES \\
\hline 18 & $\begin{array}{l}\text { Leaf damaging/ash } \\
\text { weevil }\end{array}$ & $\begin{array}{l}\text { Myllocerus } \\
\text { undecimpustulatus }\end{array}$ & Curculionidae & Coleoptera & YES & --- \\
\hline 19 & Blister beetle & Mylabris pustulatus & Meloidae & Coleptera & --- & YES \\
\hline 20 & Bud weevil & $\begin{array}{l}\text { Indozocladius } \\
\text { asperulus }\end{array}$ & Curculionidae & Coleoptera & --- & YES \\
\hline 21 & Rose ringed parakeet & Psittacula krameri & Psittacidae & Psittaciformes & --- & YES \\
\hline
\end{tabular}


Table.3 Relative abundance of insect pests under short duration pigeonpea intercropping systems during 2004 at Varanasi

\begin{tabular}{|c|c|c|c|c|c|c|c|c|c|}
\hline \multirow[t]{2}{*}{ S. No. } & \multirow[t]{2}{*}{ Insect pest } & \multicolumn{8}{|c|}{ Relative Abundance } \\
\hline & & T1 & T2 & T3 & T4 & T5 & T6 & T7 & Total \\
\hline 1 & A. crenulata & 0.34 & 0.47 & 0.60 & 0.62 & 0.67 & 0.63 & 1.00 & 4.33 \\
\hline 2 & E. kerri & 1.19 & 1.70 & 2.57 & 2.85 & 3.07 & 3.02 & 4.38 & 18.79 \\
\hline 3 & O. oneratus & 0.08 & 0.10 & 0.28 & 0.85 & 0.44 & 0.33 & 1.11 & 3.20 \\
\hline 4 & C. cribrarium & 0.17 & 0.17 & 1.29 & 0.59 & 0.95 & 0.59 & 1.51 & 5.28 \\
\hline 5 & N.viridula & 0.01 & 0.01 & 0.06 & 0.06 & 0.01 & 0.12 & 0.36 & 0.63 \\
\hline 6 & C. gibbosa & 0.22 & 0.32 & 0.47 & 0.49 & 0.47 & 0.62 & 0.82 & 3.42 \\
\hline 7 & R. pedestris & 0.09 & 0.16 & 0.26 & 0.29 & 0.31 & 0.31 & 0.52 & 1.95 \\
\hline 8 & C. cajani & 0.09 & 2.39 & 0.50 & 0.13 & 0.03 & 0.04 & 5.10 & 8.26 \\
\hline 9 & H. armigera & 0.39 & 0.66 & 0.90 & 0.91 & 0.90 & 0.81 & 1.31 & 5.86 \\
\hline 10 & G. critica & 0.20 & 0.40 & 0.58 & 0.69 & 0.62 & 0.56 & 1.01 & 4.06 \\
\hline 11 & L. boeticus & 0.12 & 0.22 & 0.31 & 0.33 & 0.33 & 0.22 & 0.53 & 2.06 \\
\hline 12 & M. testulalis & 0.17 & 0.27 & 0.43 & 0.43 & 0.40 & 0.44 & 0.72 & 2.87 \\
\hline 13 & E. atomosa & 0.16 & 0.26 & 0.35 & 0.33 & 0.32 & 0.34 & 0.62 & 2.37 \\
\hline 14 & M. undecimpustulatus & 0.15 & 0.29 & 0.41 & 0.43 & 0.55 & 0.41 & 0.86 & 3.09 \\
\hline 15 & I. asperulus & 0.13 & 0.23 & 0.30 & 0.31 & 0.33 & 0.36 & 0.62 & 2.29 \\
\hline 16 & M. pustulatus & 0.18 & 0.26 & 0.38 & 0.38 & 0.33 & 0.38 & 0.75 & 2.64 \\
\hline 17 & A. benignum & 0.10 & 0.15 & 0.26 & 0.20 & 0.20 & 0.24 & 0.52 & 1.67 \\
\hline 18 & Megachile sp & 0.03 & 0.07 & 0.12 & 0.12 & 0.12 & 0.12 & 0.17 & 0.74 \\
\hline 19 & M. usitatus & 1.78 & 2.18 & 3.00 & 3.06 & 3.35 & 3.49 & 4.68 & 21.55 \\
\hline 20 & M. obtusa & 0.40 & 0.52 & 0.72 & 0.80 & 0.80 & 0.72 & 0.99 & 4.96 \\
\hline \multicolumn{2}{|l|}{ TOTAL } & 6.00 & 10.82 & 13.80 & 13.87 & 14.21 & 13.73 & 27.56 & 100.00 \\
\hline
\end{tabular}

$\mathrm{T}_{1}=$ Pigeonpea + sorgum, $\mathrm{T}_{2}=$ Pigeonpea + bajra, $\mathrm{T}_{3}=$ Pigeonpea + greengram, $\mathrm{T}_{4}=$ Pigeonpea + cowpea, $\mathrm{T}_{5}=$

Pigeonpea + soybean, $\mathrm{T}_{6}=$ Pigeonpea + urdbean, $\mathrm{T}_{7}=$ Control $($ Sole Pigeonpea)

Table.4 Relative abundance of insect pests under short duration pigeonpea intercropping systems during 2005 at Varanasi

\begin{tabular}{|c|c|c|c|c|c|c|c|c|c|}
\hline \multirow[t]{2}{*}{ S. No. } & \multirow[t]{2}{*}{ Insect pest } & \multicolumn{8}{|c|}{ Relative Abundance } \\
\hline & & T1 & T2 & T3 & T4 & T5 & T6 & T7 & Total \\
\hline 1 & A. crenulata & 0.36 & 0.54 & 0.69 & 0.71 & 0.71 & 0.71 & 1.12 & 4.83 \\
\hline 2 & E. kerri & 0.92 & 1.61 & 2.41 & 2.53 & 2.67 & 2.77 & 4.19 & 17.10 \\
\hline 3 & O. oneratus & 0.12 & 0.17 & 0.41 & 0.63 & 0.49 & 0.62 & 1.46 & 3.90 \\
\hline 4 & C. cribrarium & 0.19 & 0.34 & 0.95 & 0.68 & 0.93 & 0.87 & 1.91 & 5.87 \\
\hline 5 & Nviridula & 0.00 & 0.01 & 0.07 & 0.07 & 0.07 & 0.08 & 0.68 & 0.98 \\
\hline 6 & C. gibbosa & 0.18 & 0.32 & 0.45 & 0.44 & 0.45 & 0.55 & 0.75 & 3.14 \\
\hline 7 & R. pedestris & 0.11 & 0.18 & 0.28 & 0.29 & 0.30 & 0.34 & 0.56 & 2.07 \\
\hline 8 & C. cajani & 0.08 & 2.30 & 0.25 & 0.18 & 0.06 & 0.11 & 3.81 & 6.79 \\
\hline 9 & H. armigera & 0.32 & 0.61 & 0.68 & 0.81 & 0.79 & 0.77 & 1.74 & 5.71 \\
\hline 10 & G. critica & 0.14 & 0.30 & 0.45 & 0.49 & 0.44 & 0.38 & 0.93 & 3.13 \\
\hline 11 & L. boeticus & 0.09 & 0.20 & 0.28 & 0.28 & 0.30 & 0.26 & 0.54 & 1.94 \\
\hline 12 & M. testulalis & 0.15 & 0.24 & 0.36 & 0.39 & 0.39 & 0.47 & 0.77 & 2.77 \\
\hline 13 & E. atomosa & 0.17 & 0.25 & 0.32 & 0.43 & 0.33 & 0.41 & 0.66 & 2.58 \\
\hline 14 & M. undecimpustulatus & 0.14 & 0.27 & 0.37 & 0.43 & 0.50 & 0.48 & 0.99 & 3.20 \\
\hline 15 & I. asperulus & 0.21 & 0.35 & 0.49 & 0.57 & 0.60 & 0.49 & 1.15 & 3.86 \\
\hline 16 & M. pustulatus & 0.16 & 0.31 & 0.41 & 0.47 & 0.38 & 0.45 & 0.79 & 2.96 \\
\hline 17 & A. benignum & 0.12 & 0.22 & 0.23 & 0.30 & 0.32 & 0.29 & 0.63 & 2.12 \\
\hline 18 & Megachile $s p$ & 0.10 & 0.15 & 0.16 & 0.19 & 0.17 & 0.19 & 0.30 & 1.27 \\
\hline 19 & M. usitatus & 1.78 & 2.20 & 2.76 & 2.88 & 3.10 & 3.25 & 4.27 & 20.26 \\
\hline 20 & M. obtusa & 0.45 & 0.67 & 0.79 & 0.88 & 0.86 & 0.77 & 1.10 & 5.53 \\
\hline \multicolumn{2}{|c|}{ TOTAL } & 5.79 & 11.24 & 12.83 & 13.67 & 13.85 & 14.29 & 28.33 & 100.00 \\
\hline
\end{tabular}

$\mathrm{T}_{1}=$ Pigeonpea + sorgum, $\mathrm{T}_{2}=$ Pigeonpea + bajra, $\mathrm{T}_{3}=$ Pigeonpea + greengram, $\mathrm{T}_{4}=$ Pigeonpea + cowpea, $\mathrm{T}_{5}=$ Pigeonpea + soybean, $\mathrm{T}_{6}=$ Pigeonpea + urdbean, $\mathrm{T}_{7}=$ Control (Sole Pigeonpea) 
Table.5 Effect of intercropping on the pod damage by gram pod borer Helicoverpa armigera in short duration pigeonpea during 2004 and 2005

\begin{tabular}{|l|}
\multicolumn{1}{|c|}{ Treatments } \\
\hline Pigeonpea + sorghum $\left(\mathrm{T}_{1}\right)$ \\
\hline Pigeonpea + bajra $\left(\mathrm{T}_{2}\right)$ \\
\hline Pigeonpea + greengram $\left(\mathrm{T}_{3}\right)$ \\
\hline Pigeonpea + cowpea $\left(\mathrm{T}_{4}\right)$ \\
\hline Pigeonpea + soybean $\left(\mathrm{T}_{5}\right)$ \\
\hline Pigeonpea + urdbean $\left(\mathrm{T}_{6}\right)$ \\
\hline Control $($ Sole pigeonpea $)\left(\mathrm{T}_{7}\right)$ \\
\hline A verage \\
\hline CD $(\mathrm{P}=0.05)$ \\
\hline
\end{tabular}

\begin{tabular}{|c|c|c|}
\hline \multicolumn{3}{|c|}{ Pod damage $(\%)$} \\
\hline $\mathbf{2 0 0 4}$ & $\mathbf{2 0 0 5}$ & Average \\
\hline $4.33(12.00)$ & $5.00(12.88)$ & $4.67(12.45)$ \\
\hline $5.67(13.76)$ & $6.00(14.15)$ & $5.83(13.96)$ \\
\hline $8.33(16.75)$ & $9.00(17.44)$ & $8.67(17.11)$ \\
\hline $5.67(13.76)$ & $5.67(13.73)$ & $5.67(13.77)$ \\
\hline $7.33(15.70)$ & $7.67(16.07)$ & $7.50(15.89)$ \\
\hline $8.00(16.41)$ & $8.00(16.41)$ & $8.00(16.41)$ \\
\hline $20.00(26.55)$ & $21.00(27.27)$ & $20.50(26.91)$ \\
\hline $8.48(16.42)$ & $8.90(16.85)$ & $8.69(16.64)$ \\
\hline 1.53 & 1.95 & 1.45 \\
\hline
\end{tabular}

Figures in parentheses are arc sin transformed values.

Table.6 Effect of intercropping on the pod damage by tur pod fly, Melanagromyza obtusa in short duration pigeonpea during 2004 and 2005

\begin{tabular}{|l|}
\multicolumn{1}{c|}{ Treatments } \\
\hline Pigeonpea + sorghum $\left(\mathrm{T}_{1}\right)$ \\
\hline Pigeonpea + bajra $\left(\mathrm{T}_{2}\right)$ \\
\hline Pigeonpea + greengram $\left(\mathrm{T}_{3}\right)$ \\
\hline Pigeonpea + cowpea $\left(\mathrm{T}_{4}\right)$ \\
\hline Pigeonpea + soybean $\left(\mathrm{T}_{5}\right)$ \\
\hline Pigeonpea + urdbean $\left(\mathrm{T}_{6}\right)$ \\
\hline Control (Sole pigeonpea) $\left(\mathrm{T}_{7}\right)$ \\
\hline A verage \\
\hline CD $(\mathrm{P}=0.05)$ \\
\hline
\end{tabular}

\begin{tabular}{|c|c|c|}
\hline \multicolumn{3}{|c|}{ Pod damage (\%) } \\
\hline $\mathbf{2 0 0 4}$ & $\mathbf{2 0 0 5}$ & Average \\
\hline $11.67(19.97)$ & $11.00(19.36)$ & $11.33(19.67)$ \\
\hline $13.33(21.40)$ & $13.00(21.12)$ & $13.17(21.28)$ \\
\hline $15.67(23.31)$ & $16.00(23.58)$ & $15.83(23.45)$ \\
\hline $15.67(23.30)$ & $16.33(23.84)$ & $16.00(13.57)$ \\
\hline $14.67(22.49)$ & $14.33(22.20)$ & $14.50(22.34)$ \\
\hline $15.67(23.31)$ & $15.33(23.04)$ & $15.50(23.18)$ \\
\hline $22.33(28.19)$ & $23.00(28.65)$ & $22.67(28.43)$ \\
\hline $15.57(23.14)$ & $15.57(23.11)$ & $15.57(23.13)$ \\
\hline 1.99 & & 1.63 \\
\hline
\end{tabular}

Figures in parentheses are arc sin transformed values.

Table.7 Effect of intercropping on the seed damage by gram pod borer Helicoverpa armigera in short duration pigeonpea during 2004 and 2005

\begin{tabular}{|l|}
\multicolumn{1}{|c|}{ Treatments } \\
\hline Pigeonpea + sorghum $\left(\mathrm{T}_{1}\right)$ \\
\hline Pigeonpea + bajra $\left(\mathrm{T}_{2}\right)$ \\
\hline Pigeonpea + greengram $\left(\mathrm{T}_{3}\right)$ \\
\hline Pigeonpea + cowpea $\left(\mathrm{T}_{4}\right)$ \\
\hline Pigeonpea + soybean $\left(\mathrm{T}_{5}\right)$ \\
\hline Pigeonpea + urdbean $\left(\mathrm{T}_{6}\right)$ \\
\hline Control (Sole pigeonpea) $\left(\mathrm{T}_{7}\right)$ \\
\hline A verage \\
\hline CD $(\mathrm{P}=0.05)$ \\
\hline
\end{tabular}

\begin{tabular}{|c|c|c|}
\hline \multicolumn{3}{|c|}{ Seed damage $(\%)$} \\
\hline $\mathbf{2 0 0 4}$ & $\mathbf{2 0 0 5}$ & Average \\
\hline $3.33(10.50)$ & $2.67(9.36)$ & $3.00(9.95)$ \\
\hline $5.00(12.88)$ & $4.33(12.00)$ & $4.67(12.47)$ \\
\hline $7.67(16.05)$ & $7.00(15.32)$ & $7.33(15.71)$ \\
\hline $7.00(15.34)$ & $6.67(14.95)$ & $6.83(15.15)$ \\
\hline $7.00(15.34)$ & $6.67(14.95)$ & $6.83(15.15)$ \\
\hline $7.33(15.70)$ & $7.33(15.70)$ & $7.33(15.71)$ \\
\hline $11.33(19.67)$ & $11.67(19.97)$ & $11.50(19.82)$ \\
\hline $6.95(15.06)$ & $6.62(14.61)$ & $6.79(14.85)$ \\
\hline 1.62 & 1.51 & 0.94 \\
\hline
\end{tabular}

Figures in parentheses are arc sin transformed values. 
Table.8 Effect of intercropping on the seed damage by tur pod fly, Melanagromyza obtusa in short duration pigeonpea during 2004 and 2005

\begin{tabular}{l}
\multicolumn{1}{|c}{ Treatments } \\
\hline Pigeonpea + sorghum $\left(\mathrm{T}_{1}\right)$ \\
\hline Pigeonpea + bajra $\left(\mathrm{T}_{2}\right)$ \\
\hline Pigeonpea + greengram $\left(\mathrm{T}_{3}\right)$ \\
\hline Pigeonpea + cowpea $\left(\mathrm{T}_{4}\right)$ \\
\hline Pigeonpea + soybean $\left(\mathrm{T}_{5}\right)$ \\
\hline Pigeonpea + urdbean $\left(\mathrm{T}_{6}\right)$ \\
\hline Control $\left(\right.$ Sole pigeonpea) $\left(\mathrm{T}_{7}\right)$ \\
\hline A verage \\
\hline CD $(\mathrm{P}=0.05)$
\end{tabular}

\begin{tabular}{|c|c|c|}
\hline \multicolumn{3}{|c|}{ Pod damage $(\%)$} \\
\hline $5.67(13.76)$ & $5.00(12.88)$ & $5.33(13.33)$ \\
\hline $6.33(14.53)$ & $6.00(14.15)$ & $6.17(14.34)$ \\
\hline $7.67(16.07)$ & $7.67(16.07)$ & $7.67(16.07)$ \\
\hline $6.33(14.57)$ & $6.67(14.95)$ & $6.50(14.76)$ \\
\hline $7.67(16.07)$ & $8.33(16.77)$ & $8.00(16.42)$ \\
\hline $8.00(16.41)$ & $8.33(16.77)$ & $8.17(16.60)$ \\
\hline $13.00(21.12)$ & $12.67(20.85)$ & $12.83(20.09)$ \\
\hline $7.81(16.08)$ & $7.81(16.06)$ & $7.81(16.07)$ \\
\hline 1.60 & 1.47 & 1.30 \\
\hline
\end{tabular}

Figures in parentheses are arc sin transformed values.

Table.9 Effect of intercropping on the grain yield of short duration pigeonpea grown under various intercropping systems during 2004 and 2005

\begin{tabular}{|l|c|c|c|}
\hline \multicolumn{1}{|c|}{ Treatments } & \multicolumn{3}{|c|}{ Grain yield $\left(\mathbf{q}\right.$ ha $\left.^{-1}\right)$} \\
\hline Pigeonpea + sorghum $\left(\mathrm{T}_{1}\right)$ & $\mathbf{2 0 0 4}$ & $\mathbf{2 0 0 5}$ & Average \\
\hline Pigeonpea + bajra $\left(\mathrm{T}_{2}\right)$ & 16.35 & 16.28 & 16.32 \\
\hline Pigeonpea + greengram $\left(\mathrm{T}_{3}\right)$ & 15.85 & 15.76 & 15.81 \\
\hline Pigeonpea + cowpea $\left(\mathrm{T}_{4}\right)$ & 15.43 & 15.37 & 15.40 \\
\hline Pigeonpea + soybean $\left(\mathrm{T}_{5}\right)$ & 16.12 & 16.04 & 16.08 \\
\hline Pigeonpea + urdbean $\left(\mathrm{T}_{6}\right)$ & 15.59 & 15.78 & 15.78 \\
\hline Control $($ Sole pigeonpea) $)$ & $\left(\mathrm{T}_{7}\right)$ & 15.58 & 15.59 \\
\hline CD $(\mathbf{P}=\mathbf{0 . 0 5})$ & 0.23 & 8.95 & 8.97 \\
\hline
\end{tabular}

The planting details

\begin{tabular}{|l|l|l|}
\hline Experimental design & $:$ & Randomized Block Design \\
\hline No. of Replications & $:$ & 3 \\
\hline Size of the plot & $:$ & $5 \mathrm{~m} \mathrm{x} 4 \mathrm{~m}$ \\
\hline Main crop and variety & $:$ & Pigeonpea, Variety UPAS-120 \\
\hline Spacing (Main crop) & $:$ & $60 \times 20 \mathrm{~cm}$ \\
\hline Intercrops and variety & $:$ & $\begin{array}{l}\text { Local varieties of sorghum, bajra, green } \\
\text { gram, cowpea, soybean, urdbean }\end{array}$ \\
\hline
\end{tabular}


Pigeonpea based intercrops and their row ratios

\begin{tabular}{|c|l|c|}
\hline Treatments Index & \multicolumn{1}{|c|}{ Crop combinations } & Row ratio \\
\hline $\mathrm{T}_{1}$ & Pigeonpea + sorghum & $1: 1$ \\
\hline $\mathrm{T}_{2}$ & Pigeonpea + bajra & $1: 1$ \\
\hline $\mathrm{T}_{3}$ & Pigeonpea + greengram & $1: 1$ \\
\hline $\mathrm{T}_{4}$ & Pigeonpea + cowpea & $1: 1$ \\
\hline $\mathrm{T}_{5}$ & Pigeonpea + soybean & $1: 1$ \\
\hline $\mathrm{T}_{6}$ & Pigeonpea + urdbean & $1: 1$ \\
\hline $\mathrm{T}_{7}$ & Control (Sole pigeonpea) & $1: 1$ \\
\hline
\end{tabular}

These results were in conformity with observation made by Mohammed and Rao (1998) who reported that pigeonpea + sorghum intercrops significantly decreased pod damage from 15.20 to $9.70 \%$ by $H$. armigera.

Singh (2002) had reported that pigeonpea sole crop showed maximum pod damage and pod damage in intercrops, pigeonpea + sorghum, pigeonpea + rice, pigeonpea + pearlmillet and pigeonpea + maize was found to be at par with each other and significantly different from sole crop.

The effectiveness of intercropping system which reduced the pod damage $(\%)$ was found to be in order of pigeonpea $+\operatorname{sorghum}\left(\mathrm{T}_{1}\right)>$ pigeonpea + cowpea $\left(\mathrm{T}_{4}\right)>$ pigeonpea + bajra $\left(\mathrm{T}_{2}\right)>$ pigeonpea + soybean $\left(\mathrm{T}_{5}\right)>$ pigeonpea + urdbean $\left(\mathrm{T}_{6}\right)>$ pigeonpea + greengram $\left(\mathrm{T}_{3}\right)>$ sole crop of pigeonpea $\left(\mathrm{T}_{7}\right)$. The pod damage by $H$. armigera in pigeonpea have been reported by Kabaria et al., (1988) 3.60 to 9.10\%, while Thakur et al., (1989) reported 12.12 to $43.70 \%$ pod damage by the pest.

The treatments, pigeonpea + sorghum $\left(\mathrm{T}_{1}\right)$ and pigeonpea + cowpea $\left(\mathrm{T}_{4}\right)$; pigeonpea + cowpea $\left(\mathrm{T}_{4}\right)$ and pigeonpea + bajra $\left(\mathrm{T}_{2}\right)$ and pigeonpea + greengram $\left(\mathrm{T}_{3}\right)$, pigeonpea + soybean $\left(\mathrm{T}_{5}\right)$ and pigeonpea + urdbean $\left(\mathrm{T}_{6}\right)$ were found to be at par with in their groups in reducing pod damage (\%) by gram pod borer in short duration pigeonpea.
Effect of intercropping on the extent of pod damage $(\%)$ by the larvae of tur pod fly (Melanagromyza obtusa) in short duration pigeonpea

The data recorded on the extent of pod damage $(\%)$ by the larvae of tur pod fly (Melanagromyza obtusa) are presented in Table 6. Pod damage (\%) was significantly reduced in pigeonpea grown under intercropping system in comparison to sole pigeonpea.

The data on pod damage (\%) by tur pod fly recorded during both the years was pooled to know the overall effect of intercropping on pod damage (\%) and it was found that pod damage (\%) was significantly reduced in pigeonpea + sorghum $\left(T_{1}\right)$ than in other treatments. The order of reduction in pod damage (\%) in intercropping system was, pigeonpea + sorghum $\left(\mathrm{T}_{1}\right)>$ pigeonpea + bajra $\left(\mathrm{T}_{2}\right)>$ pigeonpea + soybean $\left(\mathrm{T}_{5}\right)>$ pigeonpea + urdbean $\left(\mathrm{T}_{6}\right)>$ pigeonpea + greengram $\left(\mathrm{T}_{3}\right)>$ sole crop of pigeonpea $\left(\mathrm{T}_{7}\right)>$ pigeonpea + cowpea $\left(\mathrm{T}_{4}\right)$.

The treatments pigeonpea + sorghum $\left(T_{1}\right)$ and pigeonpea + bajra $\left(\mathrm{T}_{2}\right)$; pigeonpea + bajra $\left(\mathrm{T}_{2}\right)$ and pigeonpea + soybean $\left(\mathrm{T}_{5}\right)$; pigeonpea + greengram $\left(\mathrm{T}_{3}\right)$, pigeonpea + cowpea $\left(\mathrm{T}_{4}\right)$, pigeonpea + soybean $\left(\mathrm{T}_{5}\right)$ and pigeonpea + urdbean $\left(\mathrm{T}_{6}\right)$ treatments were similar with each other in their respective groups in reducing pod damage $(\%)$ in short duration 
pigeonpea. These results were in conformity with findings of Singh (2002) who reported that maximum pod damage $(\%)$ by tur pod fly was observed in pigeonpea sole crop of pigeonpea while the minimum damage $(\%)$ was recorded in pigeonpea + blackgram and pigeonpea + sorghum.

Mhase et al., (1986) noticed that M. obtusa was the most destructive on late maturing crop than the early and mid-maturing and had $61 \%$ pod damage.

Effect of intercropping on the extent of seed damage $(\%)$ by the larvae of gram pod borer (H.armigera) in short duration pigeonpea

The data recorded on the per cent seed damage by the gram pod borer $(H$. armigera) in 2004 and 2005 and pool data are presented in Table 7.

The data recorded on seed damage $(\%)$ in short duration pigeonpea in 2004 and 2005 was pooled to know the overall effect of intercropping system irrespective of years of study.

The effectiveness of intercropping system in reducing the per cent seed damage was found to be in order of pigeonpea + sorghum $\left(\mathrm{T}_{1}\right)>$ pigeonpea + bajra $\left(\mathrm{T}_{2}\right)>$ pigeonpea + soybean $\left(\mathrm{T}_{5}\right)>$ pigeonpea + cowpea $\left(\mathrm{T}_{4}\right)>$ pigeonpea + greengram $\left(\mathrm{T}_{3}\right)>$ pigeonpea + urdbean $\left(\mathrm{T}_{6}\right)>$ sole crop of pigeonpea $\left(\mathrm{T}_{7}\right)$.

Seed damage (\%) was more in pigeonpea grown alone while it was less in the intercrops pigeonpea + sorghum $\left(\mathrm{T}_{1}\right)$.

The effectiveness of intercrops in reducing seed damage $(\%)$ was statistically similar in pigeonpea + soybean $\left(\mathrm{T}_{5}\right)$, pigeonpea + cowpea $\left(\mathrm{T}_{4}\right)$, pigeonpea + greengram $\left(\mathrm{T}_{3}\right)$ and pigeonpea + urdbean $\left(\mathrm{T}_{6}\right)$.
Effect of intercropping on the extent of seed damage $(\%)$ by the larvae of tur pod fly, Melanagromyza obtusa in short duration pigeonpea

The data recorded on the per cent seed damage by larvae of tur pod fly in 2004, 2005 and pooled data are presented in Table 8 . The data on seed damage (\%) by tur pod fly in pigeonpea pertaining to 2004 and 2005 were pooled to know the accurate impact of intercrops on seed damage (\%) irrespective of years. Almost the similar trend was found in 2004 and 2005. The effectiveness of intercropping on per cent seed damage by tur pod fly larvae in short duration pigeonpea was found to be in order of pigeonpea + sorghum $\left(\mathrm{T}_{1}\right)>$ pigeonpea + bajra $\left(\mathrm{T}_{2}\right)>$ pigeonpea + cowpea $\left(\mathrm{T}_{4}\right)>$ pigeonpea + greengram $\left(\mathrm{T}_{3}\right)>$ pigeonpea + soybean $\left(\mathrm{T}_{5}\right)>$ pigeonpea + urdbean $\left(\mathrm{T}_{6}\right)>$ sole crop of pigeonpea $\left(\mathrm{T}_{7}\right)$. The treatments pigeonpea + sorghum $\left(\mathrm{T}_{1}\right)$ and pigeonpea + bajra $\left(\mathrm{T}_{2}\right)$; pigeonpea + bajra $\left(\mathrm{T}_{2}\right)$ and pigeonpea + cowpea $\left(\mathrm{T}_{4}\right)$ and pigeonpea + greengram $\left(\mathrm{T}_{3}\right)$, pigeonpea + soybean $\left(\mathrm{T}_{5}\right)$ and pigeonpea + urdbean $\left(\mathrm{T}_{6}\right)$ were found to be statistically at par with each other in their groups in reducing the per cent seed damage.

These results are in conformity with results reported by Singh (2002) who reported that minimum grain damage $(\%)$ was recorded in the intercrop + rice and was found to be at par with intercrop of pigeonpea + sorghum while sole crop of pigeonpea had the maximum grain damage (\%). Pawar (1993) noticed that pigeonpea (early) strip cropped with sorghum suffered less damage by H.armigera.

Effect of intercropping on the grain yield of short duration pigeonpea grown under various intercropping systems

The data recorded on grain yield of pigeonpea in intercropping systems are presented in Table 9. The data obtained in 2004 and 2005 
was subjected to pooled analysis to know the exact impact of intercrops on grain yield of pigeonpea. It was found that the pigeonpea grain yield was more in pigeonpea + sorghum $\left(\mathrm{T}_{1}\right)$ and less in sole crop of pigeonpea $\left(\mathrm{T}_{7}\right)$. The grain yield of pigeonpea in different intercropping systems was found to be in an ascending order of sole crop of pigeonpea $\left(\mathrm{T}_{7}\right)<$ pigeonpea + greengram $\left(\mathrm{T}_{3}\right)<$ pigeonpea + urdbean $\left(\mathrm{T}_{6}\right)<$ pigeonpea + soybean $\left(\mathrm{T}_{5}\right)<$ pigeonpea + bajra $\left(\mathrm{T}_{2}\right)<$ pigeonpea + cowpea $\left(\mathrm{T}_{4}\right)<$ pigeonpea + sorghum $\left(\mathrm{T}_{1}\right)$.

Almost similar were obtained by Singh (2002) who reported that grain infestation by podfly was maximum in sole crop of pigeonpea while pigeonpea + blackgram had the minimum grain damage. Yadav et al., (1992) reported that pigeonpea + mungbean intercrops had significantly lower grain damage by M.obtusa as compared with pigeonpea sole crop.

\section{Acknowledgements}

The first author is thankful to Institute of Agricultural Sciences, Banaras Hindu University, Varanasi for extending necessary facilities for carrying out of this research work.

\section{References}

Fager, E.W. 1957. Determination and analysis of recurrent groups. Ecology 38: 586595

Kabaria, B., Goyal, S.N. and Shah, A.H.1988. Surveillance of insect pests damage to pigeonpea at podding stage in Bharuch District, Gujarat, India. Int. Pigeonpea Newsl., 8: 15-16.

Kumar, A and Nath, P. 2004. Flower and pod pests of pigeonpea. Insect Environment, 10 (1): 12 .
Mhase, N.L., Kadam, M.V. and Ajri, D.S. 1986. Incidence of pod borer complex on early and late maturing varieties of pigeonpea. Current Research Reporter, Mahatma Phule Agricultural University, 2 (2): 312-314.

Mohammed, G. and Rao, A.S. 1998. Influence of intercrops on the incidence of Helicoverpa armigera (Hubner) in post rainy season pigeonpea. Int. Pigeonpea Newsl., 5: 46-48.

Nath, P. and Singh, A.K. 1998. Effect of intercropping of groundnut with millets and pigeonpea on the relative incidence of insect pests. Ann.Pl.Protec.Sci. 6: 151-154.

Nath, P. and Singh, R.S. 2006. Effect of biorational approaches on pigeonpea pod and grain damage by Helicoverpa armigera (Hub). Ann.of Plant Prot.Sci., 14 (1):56-61.

Pawar, C.S. 1993. Strip cropping of short duration pigeonpea with sorghum: a management option for Helicoverpa armigera. Int. Pigeonpea Newsl., 18: 33-35.

Pimental, D. 1961. Species diversity and insect population outbreaks. Ann.Entomol.Soc.Am.54: 76-86.

Rao, M.S., Reddy, K.D. and Singh, T.V.K. 2003. Impact of intercropping on Empoasca kerri of pigeonpea in rainy and post rainy season. Indian J.Ent., 65 (4):506-512.

Romeis, J, Shanower, T.G. and Zebitz, C.P.W.1998. Trichogramma egg parasitism of Helicoverpa armigera on pigeonpea and sorghum in southern India. Entomol.Exp.Appl. Submitted.

Sekhar, J.C., Singh, K.M., Singh, R.N., Singh, Y. and Malik, K.S.1997. Impact of intercropping on the incidence of green jassid, Empoasca kerri on pigeonpea. Indian J.Ent., 59 (1):119- 123.

Singh, R.S. 2002. Study on the management of insect pests associated with 
pigeonpea (Cajanus cajan (L.) Millsp.) pods through intercropping system. Ph. D. thesis submitted to B.H.U., Varanasi. Thakur, R.C., Nema, K.K. and Singh, O.P. 1989. Losses caused by pod fly (Melanagromyza obtusa Mall.) and pod borer (Heliothis armigera (Hub.) to pigeonpea in Madhya Pradesh. Bharatiya Krishi Anusandhana Patrika., 4 (2):107-111.

Vavilov, N.I.1951. The origin, variation, immunity and breeding of cultivated plants, Chronica Botanica, 13 (1/6):1366.

Wallwork, J.A.1976. The distribution and diversity of soil fauna. $355 \mathrm{p}$. Academic Press, London.

Yadav, L.S., Chaudhary, J.P. and Yadav, P.R. 1992. Effect of crop management practices on the incidence of Melanagromyza obtusa Malloch in pigeonpea. Indian J. Ent., 54 (2):109114.

\section{How to cite this article:}

Shravan Kumar, R., Paras Nath and Raju, S.V.S. 2018. Relative Abundance of Insect Pests and Pod Damage by Pod Borers in Short Duration Pigeonpea under Intercropping Systems. Int.J.Curr.Microbiol.App.Sci. 7(12): 267-280. doi: https://doi.org/10.20546/ijcmas.2018.712.033 